PROFESIONALES Y HERRAMIENTAS PARA EL DESARROLLO LOCAL Y SUS SINERGIAS TERRITORIALES. EVALUACIÓN Y PROPUESTAS DE FUTURO IX Coloquio Nacional de Desarrollo Local del GTDL-AGE 

ANTONIO MARTÍNEZ PUCHE, XAVIER AMAT MONTESINOS, ISABEL SANCHO CARBONELL y DANIEL SANCHIZ CASTAÑO (EDS.)

\section{PROFESIONALES Y HERRAMIENTAS PARA EL DESARROLLO LOCAL Y SUS SINERGIAS TERRITORIALES. EVALUACIÓN Y PROPUESTAS DE FUTURO}

IX Coloquio Nacional de Desarrollo Local del GTDL-AGE

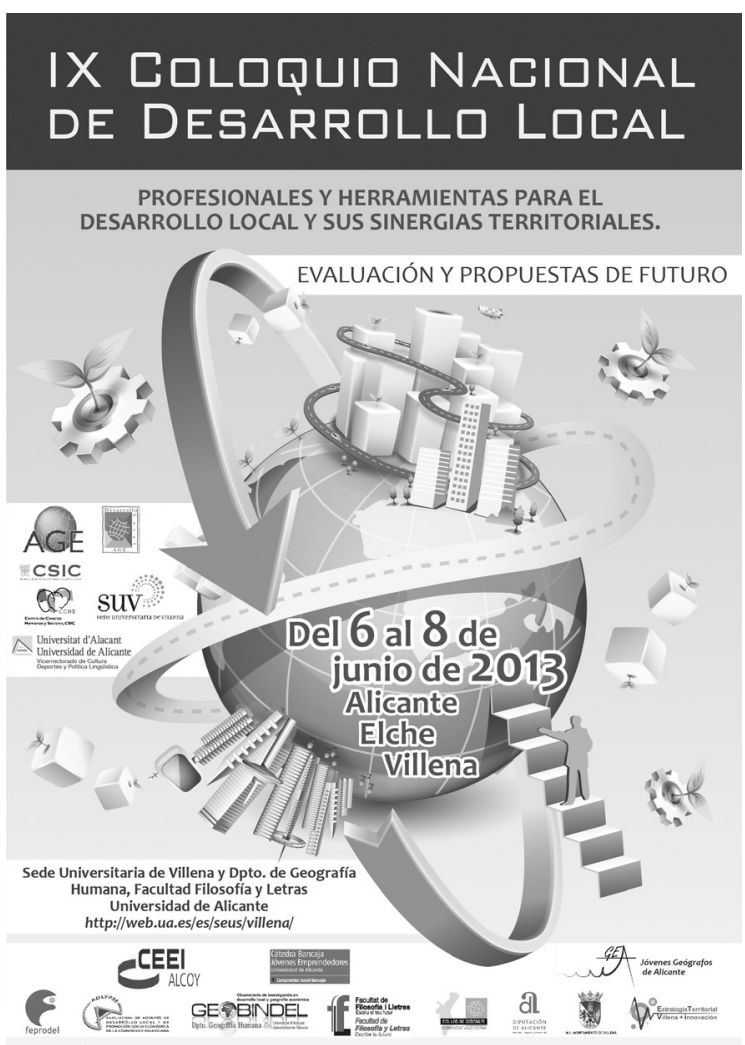


Este libro ha sido examinado y valorado por evaluadores ajenos a la Universidad de Alicante, con el fin de garantizar la calidad científica del mismo.

Publicacions de la Universitat d'Alacant

03690 Sant Vicent del Raspeig

Publicaciones@ua.es

http://publicaciones.ua.es

Telèfon: 965903480

(C) Antonio Martínez Puche, Xavier Amat Montesinos,

Isabel Sancho Carbonell y Daniel Sanchiz Castaño (eds.), 2016

(C) d'aquesta edició: Universitat d'Alacant

ISBN: 978-84-16724-00-0

Dipòsit legal: A 92-2016

Disseny de coberta: candela ink

Composició: Página Maestra (Miguel Ángel Sánchez Hernández)

Impressió i enquadernació: Guada Impresores

\section{unte \\ Unión de Editoriales
Universitarias Españolas \\ WWW.une.es
WWA}

Esta editorial es miembro de la UNE, cosa que garantiza la difusión y comercialización nacional y internacional de sus publicaciones.

Reservados todos los derechos. Cualquier forma de reproducción, distribución, comunicación pública o transformación de esta obra sólo puede ser realizada con la autorización de sus titulares, salvo excepción prevista por la ley. Diríjase a CEDRO (Centro Español de Derechos Repográficos, www.cedro.org) si necesita fotocopias o escanear algún fragmento de esta obra. 


\title{
EVALUACIÓN Y POSIBLES ALTERNATIVAS AL PLAN INTEGRAL ZONA NORTE DE ALICANTE: EFECTOS Y CONSECUENCIAS EN EL DESARROLLO LOCAL DEL BARRIO JUAN XXIII $2^{\circ}$ SECTOR
}

\author{
Javier Ortega Fernández \\ Alumno del máster en Desarrollo Local e Innovación Territorial \\ jof4@alu.ua.es
}

\section{RESUMEN}

Tras la implantación del Plan Integral barrios Zona Norte de Alicante durante el periodo 2007-2013, hemos realizado una investigación enfocada al conocimiento, evaluación y análisis de los efectos sociales y económicos que ha supuesto el desarrollo del Plan. Contextualizamos el estudio en el barrio Juan $X X I I I-2^{\circ}$ Sector de Alicante, ya que este espacio corresponde a uno de los seis barrios objeto del Plan y, además, es uno de los más vulnerables de la ciudad. A su vez, enfatizamos nuestro análisis en dos ejes temáticos: formación para el empleo y participación ciudadana (dos temáticas características del desarrollo local). Utilizamos aquellos instrumentos metodológicos que nos permiten reflexionar, pensar y debatir, y así confeccionar una investigación que adopte un enfoque crítico. Son los propios agentes sociales y técnicos, relacionados directamente con el Plan, los que nos muestran su visión del mismo y nos permiten articular una evaluación de sus efectos a nivel social, económico e, incluso, político.

Palabras Clave: Plan Integral Zona Norte, Barrios Marginales, Desarrollo Local, Participación Ciudadana, Actividad Comercial.

EVALUATION AND POSSIBLE ALTERNATIVES TO COMPREHENSIVE PLAN NORTH OF ALICANTE: EFFECTS AND CONSEQUENCES IN THE LOCAL DEVELOPMENT SECTOR OF 2nd QUARTER JUAN XXIII 


\section{ABSTRACT:}

After the implementation of the Comprehensive Plan of north Alicante neighborhoods during the period 2007-2013, we performed an investigation focused on knowledge, assessment and an analysis of social and economic effects has led to the development of the Plan.

We contextualize the study in the Juan XXIII-2nd Sector of Alicante, as this space corresponds to one of the six districts under the Plan and also is one of the most vulnerable in the city. At the same time, we emphasize our analysis on two themes: employment training and citizen participation (two thematic characteristics of local development). We use those methodological tools that allow us to reflect, think and discuss, and so make an investigation to take a critical approach. It is the social and technical agents themselves, directly related to the Plan, who show us their vision of it and allow us to articulate an assessment of its effects on social, economic and even political level.

Key words: Plan Integral Zona Norte, slums neighborhoods, local development, public participation, comercial activity.

\section{INTRODUCCIÓN}

En la presente investigación trataremos de indagar y evaluar qué efectos ha tenido la implantación de un Plan Integral que se viene desarrollando desde 2007 en los barrios catalogados como Zona Norte de Alicante (Virgen del Remedio, Colonia Requena, Cuatrocientas Viviendas, Virgen del Carmen, Sidi Ifni Nou Alacant, Juan XXIII $2^{\circ}$ Sector), el cuál plantea como principales objetivos mejorar las condiciones sociales, culturales, educativas, económicas, de infraestructuras y de seguridad de los barrios.

Para ello desarrollamos un trabajo de investigación, estructurado a partir de un marco teórico donde realizaremos una transición teórica de lo general a lo concreto. Dentro de la parte más global hacemos referencia a aquellos conceptos y acepciones que afectan directamente a los barrios objeto de estudio (exclusión social, barrios marginales, comunidad, guetos residenciales). Se trata de desarrollar una visión general de todas aquellas características que afectan a los barrios en cuestión y que desde diversas disciplinas de las ciencias sociales se ha dedicado una parte importante del trabajo académico a lo largo de los años. Un conocimiento profundo y bibliográfico de dichas cuestiones conlleva una mayor riqueza epistemológica acorde con la temática que nos ocupa. Antes de comenzar con el trabajo de campo es fundamental que expongamos un diagnóstico socio-demográfico y económico del barrio sobre el que vamos a trabajar, de esta forma detallaremos cuál es la situación espacial, social y económica del mismo. 
La investigación parte de dos hipótesis donde se cuestiona la consecución de una serie de objetivos que se plantean en el Plan en los ejes temáticos de: Inserción laboral y Formación, y Participación Ciudadana. Las hipótesis de trabajo nos surgen a partir de una serie de cuestiones previas al desarrollo de la investigación y, a su vez, servirán para marcarnos todo el proceso metodológico y analítico del mismo. Algunas de ellas son: ¿existe una implicación real por parte de la Administración Pública en la implantación y seguimiento del Plan?; ¿se ha elaborado el plan desde una visión prospectiva?; ¿se han conseguido los objetivos esperados y plasmados en el Plan?; ¿sería posible la aplicación del Plan sin la adquisición de ayudas públicas europeas (programa de ayuda Iniciativa URBAN)?, ¿se ha tenido en cuenta a la población residente en los barrios Zona Norte de Alicante para la elaboración del Plan?, ¿de qué forma? Nuestro estudio tratará de dar respuestas a todas estas cuestiones y además plantear un escenario crítico y reflexivo sobre la temática que nos ocupa, a través de entrevistas semi-estructuradas sobre agentes sociales y técnicos relacionados directamente con el desarrollo del Plan Integral Barrios Zona Norte.

\section{CONTEXTOS DE EXCLUSIÓN SOCIAL, BARRIADAS MARGINALES Y COMUNIDAD}

Algunos autores hacen referencia al concepto de exclusión social como la falta de participación de los individuos en las relaciones sociales en las que se producen los bienes sociales necesarios para la satisfacción de las necesidades humanas (Paugman, 1996). En algunas ocasiones la exclusión nos ayuda a entender la pobreza y a comprenderla en un sentido más amplio, "el término exclusión puede entenderse como un concepto multidimensional de la pobreza que introduce aspectos de participación social y de realización de derechos como seguridad física, de sustento y de protección ante contingencias, identidad y plena ciudadanía" (Martínez Román, 2001: 71). El contexto espacial y socio-económico también es muy importante cuando tratamos de analizar la exclusión social, por ello podemos identificar, según Castel (1995), tres espacios sociales en relación a la exclusión social. Las zonas se clasifican según el grado de exclusión de menor a mayor: zona de integración, seguridad o estabilidad; zona de vulnerabilidad, precariedad o inestabilidad (zonas con elevado riesgo de exclusión social); y zona de exclusión o marginación.

La planificación urbanística ha permitido diversificar las ciudades a través de barrios residenciales, con características sociales y económicas diferenciadas, al mismo tiempo que se produciría una serie de asentamientos que reunían las condiciones físicas, arquitectónicas y sociales para el abandono, la desatención y la exclusión social. Ante esta situación, según señala Gomez Gil, aparecen en las ciudades territorios considerados como el refugio de los perdedores, de los excluidos, de los que no son capaces de triunfar en una sociedad cada vez más competitiva, o simplemente de sectores en situación 
cada vez más precaria o con trabajos mal remunerados (Gómez, 2006). Se construyen "fronteras ficticias" entre algunos barrios en decadencia y el resto de asentamientos residenciales, como nos indica Friedman, "algo que no está ocurriendo es la desaparición de las fronteras. Antes bien, parece que se están levantando en cada nueva esquina de cada calle de todos y cada uno de los barrios en decadencia de nuestro mundo" (Friedman, 1999: 239).

¿La "comunidad" es una característica obsoleta en las sociedades postmodernas? En primer lugar sería conveniente exponer que entendemos por сотиnidad, para ello debemos hacer referencia preferentemente a conceptos como hedonismo o individualismo, pautas de comportamiento cada vez con más presencia en la sociedad postmoderna. La pérdida de vínculos sociales y personales entre vecinos que habitan a escasos metros de sus respectivos hogares conlleva un grave problema, sobre todo, para aquellos colectivos más vulnerables y con menos recursos. Las fuerzas globales, el individualismo exacerbado y las estrategias de desvinculación de los poderes globales nos llevan, según Bauman, a que "cuanto más pulverizados estén, cuanto más débiles y exiguas las unidades en las que estén divididos, tanto más disiparán su ira en la lucha contra los vecinos de al lado, parecidamente impotentes, y menor será la probabilidad de que actúen conjuntamente alguna vez" (Bauman, 2003: 101). Muchas minorías étnicas tienen todavía muy arraigada la necesidad cultural de hacer "comunidad", de mantener lazos personales estrechamente unidos y, de esta forma, mantener una resistencia que no sería posible sin la ayuda mutua entre iguales. Allí donde el Estado no puede llegar, debe ser la comunidad la que provee a los ciudadanos las necesidades sociales que tienen los individuos. En muchas ocasiones, el término de comunidad tiene una acepción negativa, especialmente cuando se da en colectivos que confunden la comunidad con un ente cerrado, localista y excluyente. En estos casos un exceso de la mal comprendida comunidad desencadena en un incremento de la exclusión social.

\section{Características SOCIO-DEMOgráficas del barRio JuAn XXIII-2 ${ }^{\circ}$ SeCtor (Ba- rrio de la Zona Norte de Alicante)}

Con la finalidad de delimitar el ámbito de estudio trataré de realizar un análisis cuantitativo y descriptivo de la situación sociodemográfica del barrio Juan XXIII $2^{\circ}$ Sector (Alicante), de esta forma acotamos la investigación a un barrio en concreto del término municipal de Alicante. Se utilizarán fuentes secundarias, de carácter estadístico, como es el Padrón Municipal (a través de su búsqueda en el Instituto Nacional de Estadística -INE-) ya que nos permite obtener datos poblacionales por secciones censales y, a su vez, nos da la posibilidad de conseguir información territorial por barrios.

El barrio Juan XXIII-2 ${ }^{\circ}$ Sector pertenece al término municipal de Alicante, situado al norte de la ciudad. Está delimitado, al norte, con los barrios de 
Villafranqueza, al sur, con los barrios de Garbinet y Vistahermosa, y, al oeste, con los barrios de Colonia Requena, Virgen del Remedio y Cuatrocientas viviendas (estos tres también incluidos en el Plan Integral Barrios Vulnerables Zona Norte).

La población total que compone el barrio es, a fecha de 1 de enero de 2012, de 10.987 habitantes, en relación al conjunto del término municipal de Alicante supone un 3,28\% de la población. La distribución por sexo, como podemos comprobar, no es significativa ya que existen escasas diferencias cuantitativas entre el total de hombres y mujeres que forman parte de este ámbito territorial, representan un total de 5.621 hombres y 5.366 mujeres, respectivamente (observamos una Sex Ratio $^{1}$ de 105 hombres por cada 100 mujeres). En el barrio Juan XXIII-2 ${ }^{\circ}$ Sector y según el Índice de Juventud ${ }^{2}$ para el año 2012, hay 162 jóvenes por cada 100 viejos, mientras que en la ciudad de Alicante, para ese mismo año, son 118 jóvenes por cada 100 viejos que viven en la ciudad. La diferencia es significativa y nos permite hacer valoraciones sobre la estructura de edad del barrio objeto de estudio. Estamos ante un espacio residencial muy joven respecto al conjunto de la ciudad, por lo tanto los más jóvenes ocuparan un papel muy relevante en el desarrollo integral del barrio.

Es importante destacar la elevada presencia de colectivos de inmigrantes residentes en el barrio. El 23\% de vecinos y vecinas son de origen extranjero, este hecho explica a su vez las diferencias de estructura poblacional que se dan entre el propio barrio y el resto de la ciudad. Los flujos migratorios se convierten en un fenómeno social muy presente en el territorio analizado y condiciona, a su vez, las interrelaciones sociales que se producen dentro del mismo. Para la ciudad de Alicante el total de extranjeros es del $15 \%, 8$ puntos por debajo del total de extranjeros residentes en el barrio Juan XXIII. La gran mayoría de inmigrantes son de origen africano, casi la mitad (43\%) provienen de este continente (principalmente de Argelia, Marruecos y Senegal, respectivamente) con culturas, costumbres y lenguas distintas a la autóctona. En su mayoría lo hacen con escasos recursos y la problemática se acrecienta todavía más por las limitaciones que conlleva la diferenciación cultural y de lenguaje en la inclusión social de estos colectivos.

Otro de los colectivos con elevada presencia en el barrio es la población de etnia gitana. Carecemos de información estadística que nos permita identificar

1 La Sex Ratio o Índice de Masculinidad es un indicador demográfico que nos permite constatar el número de hombres que hay en un territorio por cada 100 mujeres. Para ello se divide el total de hombres de un territorio determinado por el total de mujeres, y el resultado final lo multiplicaríamos por 100.

2 El Índice de Juventud es un indicador demográfico que mide el número de jóvenes entre 0 y 19 años (en función de las referencias bibliográficas el intervalo de edad varía y mucho autores consideran a los jóvenes entre 0 y 16 años) por cada 100 personas viejas de más de 65 años. Este índice nos permite conocer el grado de juventud persistente en relación a las capas de mayor edad de la población. 
y/o cuantificar la composición y distribución de la población en función de la etnia de pertenencia, ya que los indicadores oficiales no especifican la pertenencia o no a una distinta etnia. El trabajo de observación que hemos realizado nos ha permitido reconocer la elevada presencia de este tipo de población, sin incidir en sus relaciones sociales, culturales y políticas.

\section{Síntesis del plan integral barrios vulnerables Zona Norte de Alicante}

El Ayuntamiento de Alicante decide en 2004 (año en el que comienza la elaboración y estructuración del Plan), frente a la situación de precariedad social, económica y ambiental que sufrían diferentes barrios de la ciudad, impulsar y promover un Plan Municipal para aquellas zonas de la ciudad que reunían unas características sociales y económicas similares. Se decidió elaborar un Plan de carácter integral, a través de la elaboración de programas y actuaciones de diferente temática, con el fin de mejorar las condiciones sociales, económicas y ambientales desde un enfoque transversal. los barrios sobre los que el presente plan actúa son los siguientes: Virgen del Remedio, Colonia Requena, Virgen del Carmen, Juan XXIII $2^{\circ}$ Sector, Cuatrocientas Viviendas y Sidi-Ifni Nou Alacant. Son 6 barrios del término municipal de Alicante (se les denomina Barrios Zona Norte) y reúnen características similares, entre ellas: fuerte índice de desempleo, débil tasa de actividad económica, alto nivel de pobreza y exclusión, elevado número de inmigrantes y minorías étnicas, bajo nivel educativo, nivel elevado de criminalidad y delincuencia, y degradación ambiental de la zona ${ }^{3}$.

La temporalidad del plan abarca un periodo que transcurre de 2007 a 2013, es decir 6 años en los que se presentaran diferentes programas y actuaciones y se destinará un presupuesto concreto para cada año. Para conocer con mayor profundidad la estructura del plan es conveniente que hagamos una breve clasificación de los ejes y estrategias que sigue el presente plan que estamos describiendo (Plan Integral Barrios Zona Norte de Alicante, 2007):

Tabla 1 Clasificación de los Ejes estratégicos del Plan Integran Barrios Zona Norte de Alicante

\begin{tabular}{|c|c|c|c|c|}
\hline $\begin{array}{l}\text { Hacer un barrio } \\
\text { físicamente } \\
\text { atractivo e } \\
\text { integrado con el } \\
\text { resto de la ciudad }\end{array}$ & $\begin{array}{c}\text { Formación } \\
\text { para el Empleo } \\
\text { y Fomento } \\
\text { del Espíritu } \\
\text { Empresarial }\end{array}$ & $\begin{array}{l}\text { Seguridad } \\
\text { ciudadana }\end{array}$ & $\begin{array}{c}\text { Inserción } \\
\text { Socioeducativa, } \\
\text { Familiar y Salud } \\
\text { Comunitaria }\end{array}$ & $\begin{array}{c}\text { Integración } \\
\text { cultural, } \\
\text { Convivencia y } \\
\text { Participación } \\
\text { Ciudadana }\end{array}$ \\
\hline
\end{tabular}

Fuente: Plan Integral Barrios Vulnerables Zona Norte de Alicante

3 Ver Plan Integral Barrios Vulnerables Zona Norte de Alicante (2007). 
El presente plan se financia a través del Fondo Europeo de Desarrollo Regional (FEDER), concretamente a partir un programa de ayuda que impulsa la Comunidad Europea que recibe el nombre de iniciativa URBAN ${ }^{4}$, corresponde al periodo 2007-2013. Se trata de financiación a escala comunitaria destinada a ciudades y áreas urbanas con el fin de favorecer el desarrollo local desde un enfoque integrado y, por tanto, no sólo favorecer el crecimiento y el empleo, sino también perseguir objetivos sociales y medioambientales. Para este periodo se ha presupuestado sobre el conjunto del Plan una cuantía total de 167.708.5985. En relación al objeto estratégico de la investigación sería conveniente que destacásemos la cuantía destinada a Participación Social, con un total de 3.200.350 Euros (supone menos de un 2\% del total del presupuesto del Plan; y la cuantía que se destina al área temática de Formación e Inserción Laboral (5.183.882 euros, poco más el 3\% del total presupuestado) y a la de Desarrollo del tejido económico apoyando la actividad comercial (1.905.063 euros, poco más de $1,1 \%$ del presupuesto total).

\section{Metodología de La inVestigación}

Tras la revisión bibliográfica y descripción de los datos estadísticos más relevantes para esta investigación, la metodología aplicada del estudio consistirá en la elaboración e implementación de técnicas de investigación social cualitativas, en concreto, entrevistas semi-estructuradas dirigidas a agentes sociales que tienen estrecha relación en el desarrollo del Plan Integral Barrios Vulnerables de la Zona Norte de Alicante. Las cuestiones están abiertas a respuestas con un marcado sesgo de subjetividad, por consiguiente hemos escogido una muestra heterogénea en tanto en cuanto tienen funciones diversas en los barrios de la Zona Norte. Las organizaciones sociales y/o organismos técnicos a los que le hemos realizado las respectivas entrevistas son: la AAVV Juan XXIII, la Fundación del Secretariado Gitanon (FSC), el colectivo de Jóvenes Solidarios, y 2 Técnicos del Centro de Coordinación del Plan Zona Norte.

4 Un total de 43 ciudades españolas (entre ellas la ciudad de Alicante) han sido seleccionadas en el programa Iniciativa URBAN 2007-2013, con el fin de impulsar proyectos de desarrollo urbano, integral y sostenible. La cuantías correspondientes (344,66 millones de euros para el total de ciudades españolas) proceden de los Fondos Europeos de Desarrollo Regional (FEDER). Los proyectos financiados deberán tener un carácter integral, atendiendo al desarrollo de un conjunto multidisciplinar de actuaciones para hacer frente a los problemas de una zona urbana seleccionada dentro del municipio y con una clara desventaja social y económica respecto al conjunto del mismo.

5 Ver el Epígrafe 7 del Plan Integral Barrios Vulnerables Zona Norte de Alicante, donde se detalla el presupuesto destinado a cada plan de acción, por área temática y por actuaciones concretas. 


\section{Análisis cualitativo del Plan Integral Zona Norte}

\subsection{Elaboración y coordinación del Plan}

La estructuración del Plan Integral Barrios Vulnerables de la Zona Norte se empezó a desarrollar a partir de 2004. Fue, en gran medida, un trabajo puramente técnico, sin embargo la estructura y contenido del Plan no cuenta con una participación exclusivamente técnica, ya que se concedió la oportunidad a las diferentes entidades sociales que trabajaban activamente en los barrios de la Zona Norte de participar directamente en la elaboración y estructuración de los proyectos, actividades e iniciativas del Plan.

"Cuando se empezó a elaborar el Plan se hicieron estudios bastante exhaustivos que fueron muy participativos. Se realizaron talleres participativos donde salieron mucho de los proyectos, en función de las necesidades que creían necesario las entidades sociales". (TÉCNICO DEL PLAN)

Podemos identificar diferentes puntos de vista muy críticos respecto a la coordinación entre Áreas del Ayuntamiento por parte de entidades sociales como "Jóvenes Solidarios" y la "AAVV de Juan XXIII", convirtiéndose en el principal obstáculo para poner en marcha los proyectos, iniciativas o programas que conforman el Plan. Desde "Jóvenes Solidarios" afirman lo siguiente:

"El Plan ha obligado a trabajar conjuntamente diferentes áreas, aunque es muy difícil, no ha existido coordinación por parte del Ayuntamiento en general, a nivel ciudad”. (JÓVENES SOLIDARIOS)

No es baladí que algunas entidades sociales consideren la descoordinación entre áreas como una de las principales limitaciones en el desarrollo de los proyectos. Esto se traduce a partir de las Comisiones de Acción ${ }^{6}$. La realidad, actualmente, es que llevan más de 1 año y medio sin convocarse ${ }^{7}$, mientras las Comisiones son un instrumento importante en la coordinación de áreas y en la participación de las entidades sociales en la ejecución y evaluación de proyectos; por consiguiente, el hecho de que no exista voluntad política para convocar este tipo de comisiones está perjudicando gravemente el desarrollo participativo del Plan Integral de la Zona Norte.

6 Órgano que depende directamente de la "Comisión Técnica Institucional" (órgano que funciona a nivel político), las cuales las conforman representantes políticos, técnicos institucionales, entidades y asociaciones. Las Comisiones de Acción se organizan por distintas áreas que configuran el Plan Integral, son en definitiva, grupos de trabajo por áreas donde las entidades sociales participan activamente en el desarrollo del plan y se construyen los espacios para desarrollar una coordinación más eficiente.

7 A fecha 17 de agosto de 2013. 
6.2. Objetivos del eje estratégico de "Formación para el Empleo" y "Fomento del Espíritu Empresarial"

\section{Promoción de la actividad comercial}

El fomento de la actividad comercial como uno de los objetivos principales del eje de Formación para el Empleo y Fomento del Espíritu Empresarial, ha sido al que mayor atención le han dedicado los entrevistados. Muchos muestran una valoración positiva respecto a los proyectos o programas enfocados a la promoción de la actividad comercial, sin embargo, ¿cuáles han sido los efectos por ejemplo en barrios como Juan XXIII- $2^{\circ}$ Sector? La situación comercial para el barrio en cuestión, según apuntan Jóvenes Solidarios, es que no se ha creado ningún comercio nuevo. Todos los entrevistados coinciden que la causa principal del deterioro progresivo del pequeño y mediano comercio se debe a la instalación de una gran superficie comercial en el mismo barrio.

"Se ha puesto en marcha un "Mercadona" que ha terminado de ahogar al pequeño comercio". (TÉCNICO DEL PLAN)

El resultado final respecto al desarrollo de la actividad comercial se ha monopolizado en una única superficie comercial, degradando el tejido comercial de la zona y, por consiguiente, frenando el desarrollo comercial y económico de los vecinos y vecinas del barrio. En lo que respecta a las entrevistas formuladas hemos podido observar posturas enfrentadas frente a este hecho, justificando muchos de ellos la nueva superficie comercial como elemento facilitador de la vida de los vecinos y vecinas del barrio.

Se puede considerar, por tanto, que existen iniciativas antagónicas para fortalecer y promocionar la actividad comercial de los barrios económicamente más degradados. El caso de Juan XXIII es un hecho que clarifica esta argumentación. En esta zona sí que se ha apostado por favorecer la actividad comercial, aunque esta acción la ha monopolizado un único comercio.

\section{Coyuntura de crisis económica y limitaciones del Plan}

Para la mayoría de entrevistados la coyuntura de crisis económica no sólo ha afectado considerablemente el desarrollo de los objetivos en el área de Empleo, Formación y Actividad Comercial, sino que la consideran el motivo principal por el cual los programas y proyectos estructurados en el Plan no ha conseguido lograr la integración socio-laboral de los sectores de la población con mayores dificultades, potenciar la igualdad de oportunidades en el ámbito laboral y promocionar la actividad comercial.

Otro de los obstáculos y justificaciones que muestran los técnicos de coordinación del plan y diversas entidades sociales es el insuficiente contenido de los proyectos de empleo para ser generadores de actividad laboral. Los técnicos 
de coordinación del Plan son muy conocedores de donde están los límites hasta donde pueden llegar las acciones de empleo proyectadas y ejecutadas, de esta forma, podemos considerarlos como proyectos insuficientes que abarcan a un escaso número de población de la Zona Norte y sus resultados escapan en gran medida de objetivos demasiado ambiciosos.

Los diversos actores entrevistados señalan que están trabajando con iniciativas, programas y actividades que no tienen la potestad funcional para que se cumplan tales objetivos. Esta justificación nos presenta la siguiente cuestión, ¿estamos ante objetivos demasiado ambiciosos para un Plan Integral de estas características?

\subsection{Objetivos del eje estratégico de "Participación Ciudadana"}

Espacios para la participación en la Zona Norte

El Plan ha conseguido crear espacios donde los residentes de la Zona Norte puedan acudir y tener la posibilidad de tramitar quejas, buscar información y demás actividades que faciliten la participación de los residentes en sus barrios. Desde la Concejalía de Participación Ciudadana, se ha dotado a estos barrios de los equipamientos necesarios para la participación donde actúan conjuntamente técnicos, entidades sociales y, en ocasiones, mediadores sociales. Aunque, ¿es suficiente con crear espacios para la participación social para fomentar el tejido social y participativo del barrio? Nosotros planteamos la siguiente reflexión al respecto ya que la dotación de infraestructura no garantiza la aplicación de las mismas, por ello creemos necesario realizar un análisis al respecto que trate de visibilizar el desarrollo del tejido asociativo que se ha tratado de impulsar y fomentar desde el Plan que estamos investigando.

\section{Incentivar el tejido asociativo en la Zona Norte}

El Plan tiene programado una convocatoria de subvenciones para toda aquella organización social sin ánimo de lucro que desee llevar a cabo actividades en el barrio. Ante este hecho, aparentemente beneficioso para los barrios de la zona norte, se presenta una peyorativa a raíz de la siguiente afirmación desde Jóvenes Solidarios:

"Las subvenciones han hecho de "efecto llamada" ya que han presentado solicitud muchas entidades sociales que nunca antes habían realizado ninguna actividad en los barrios de la zona Norte". (JÓVENES SOLIDARIOS)

Ante la escasa financiación que reciben las asociaciones, fundaciones $\mathrm{u}$ otras organizaciones sin ánimo de lucro a raíz de la crisis económica y del sector público cualquier convocatoria de subvención sería bien acogida por las 
entidades sociales. Este hecho no tiene por qué ser, a priori, perjudicial para los barrios de la Zona Norte, el problema sería susceptible de surgir cuando se dejen de convocar subvenciones para procesos de actuación en estos espacios. Por ello, planteamos la siguiente reflexión: ¿seguirán actuando sobre la Zona Norte aquellas entidades sociales que no lo habían hecho con anterioridad y que no pueden acogerse a este tipo de ayudas que les "obligan" a trabajar dentro de un determinado espacio territorial? Respecto a este contexto, los técnicos del equipo de coordinación de proyectos del Plan niegan esta situación y creen, unánimemente, que este hecho no se ha dado y únicamente se ha producido en casos puntuales.

\subsection{La programación de una $2^{a}$ fase del Plan Integral: efectos y consecuencias}

Se diseñó con el objeto de realizar una $2^{\mathrm{a}}$ fase de un periodo quinquenal. De esta forma, muchos de los objetivos que se diseñaron para la $1^{\text {a }}$ fase tendrían mayor probabilidad de cumplirse o de acercarse en mayor medida a ellos. Un Plan de las característica de este, con el elevado número de proyectos holísticos e integrales, puede quedarse en la cuneta o desintegrarse si no se consigue la financiación adecuada para promover una $2^{\mathrm{a}}$ fase.

¿Qué consecuencias puede tener sobre la población de la Zona Norte la no programación de una $2^{\text {a }}$ fase del Plan? Todos los entrevistados son conscientes de que se producirán efectos negativos sobre los vecinos y vecinas de dicha zona, y sobre el funcionamiento pro-activo de muchas entidades sociales. Según señalan algunas de las organizaciones sociales entrevistadas, los colectivos que más lo notarán serán aquellas familias que han hecho un uso directo de todos los programas, actividades y proyectos del Plan.

"A nivel de convivencia (...) sí que se va a notar, de tener un montón de trabajadores sociales, de educadores, de sociólogos trabajando en la zona, trabajando por la buena convivencia, por los chavales para que tengan recursos después de ir al cole, que vayan al cole, técnicos que trabajan el absentismo; esos recortes sí que se van a notar y entonces va a haber un montón de gente que diga: oye si soy racista soy racista; el nivel de crispación aumentará seguro". (JÓVENES SOLIDARIOS)

\section{Conclusiones}

Uno de los objetos principales por los que se elabora esta investigación es alcanzar la información cualitativa suficiente que nos permita reflexionar sobre el modo de actuar de las administraciones locales en barrios marginales y vulnerables. La hipótesis de la investigación está enfocada a la consecución o no de los objetivos que el Plan Integral de la Zona Norte se plantea en los ejes estratégicos de Empleo y Participación Ciudadana. Podemos concluir, por tanto, que la hipótesis ha servido para analizar los objetivos desde diferentes 
perspectivas y conocer, a su vez, en qué grado se han podido cumplir esos objetivos y cuáles han sido los principales motivos, según los entrevistados, de la no consecución total de los objetivos planteados.

¿Podríamos catalogar el Plan Integral de los barrios de la Zona Norte como un instrumento para el fomento del "Desarrollo Local"? Sí que podríamos considerar todos los programas e iniciativas como elementos representativos de "desarrollo" de un territorio. También se puede catalogar como integral ya que actúa sobre diferentes áreas temáticas interrelacionadas, a su vez, con el desarrollo social, ambiental y económico de los barrios. Aunque esta caracterización de Plan Integral y Holístico es susceptible de ambigüedad y escepticismo si atendemos a los resultados de la investigación. A continuación se plantean una serie de cuestiones que nos permitirán reflexionar sobre la condición de "Integral" y de "Desarrollo Local" del presente Plan que hemos analizado:

- ¿Se puede considerar una actuación "Integral" cuando existentes deficiencias en la coordinación entre diferentes áreas temáticas?

- ¿Se actúa dentro del marco del "Desarrollo Local" cuando se plantean una serie de objetivos muy difíciles de alcanzarse del el ámbito local?

- ¿Cuándo se apuesta por el "Desarrollo Local" de un territorio, cabe la posibilidad de abandonar lo que se había hecho hasta entonces y no asumir la graves consecuencias que supondría no continuar con el mismo?

Estructurar un Plan de estas características, en ocasiones nos sugiere si realmente estamos ante políticas locales que buscan mejorar las condiciones sociales, económicas y ambientales de barrios marginales o, por el contrario, se realizan planes de estas características aprovechando la financiación europea con el fin de "lavar la imagen" de las políticas locales segregacionistas sobre los colectivos más vulnerables de la ciudad.

El presente estudio no se realiza con el fin de alcanzar conclusiones objetivas y concretas. Estamos ante una investigación abierta, expuesta a un marco reflexivo con el fin de favorecer posteriores estudios sobre esta misma línea de investigación.

\section{BIBLIOGRAFÍA}

BAUMAN, Zygmunt (2003). Comunidad. En busca de seguridad en un mundo hostil; Siglo XXI, Madrid (España).

BAUMAN, Zygmunt (2007). Tiempo líquidos; Tusquets Editores, Barcelona (España).

BECK, Ulrich (2002). La sociedad del riesgo global; Siglo XXI, Madrid (España).

BOOKCHIN, M. (1991). The Ecology of Freedom. The emergence and dissolution of hierarchy; Black Rose, Montreal. 
BOTERO, Restrepo (2007). "Relaciones Estado-sociedad civil en el campo social. Una reflexión desde el caso colombiano", Revista del CLAD Reforma y Democracia, No. 7, Caracas.

CASTEL, R. (1997 [1995]) La metamorfosis de la cuestión social. Una crónica del salariado. Paidós: Argentina.

DELGADO PÉREZ, G.J. (2007): La participación en el planeamiento urbanístico y el fenómeno de la teledetección. Geocrítica, Vol. XI. Núm. 245 (12)

DENCH, Geoff (1975). Maltese in London: a Case-Study in the Erosion of Ethnic Consciousness; Routledge and Kegan Paul, Londres.

DÍEZ HOCHLEITNER, Ricardo (2006). Prólogo, en Meadows, Donella; Randers, Jorgen; Meadows, Dennos. Los límites del crecimiento. 30 años después; Galaxia Gutenberg/Círculo de Lectores, Barcelona.

DOYAL, L., GOUGH, I. (1994) Teoría de las necesidades humanas. Madrid: Fuhem.

EDWARDS, Brian y HYETT, Paul (col.) (2004). Guía básica de la sostenibilidad. Barcelona: Gustavo Gili, p. 105.

FERULLO, H. (2006). "El concepto de pobreza en Amartya Sen", en Revista Valores en la Sociedad Industrial, No. 66, Agosto, pp. 10-16.

GARCÍA DE ENTERRÍA, Eduardo y PAREJO ALFONSO, Luciano (1981). Lecciones de derecho urbanístico; Civitas, Madrid.

GÓMEZ GIL, Carlos (2008). "Inmigración, urbanismo y cambios socioterritoriales en la ciudad de Alicante", en Segrelles Serrano, José Antonio (Coord.) Inmigración, Sociedad, Economía: ¿Oportunidad o Problema? Instituto alicantino de cultura "Juan Gil-Albert", Alicante, pp. 73-102.

GRUSKY, David (2001). Social Stratification: Class, Race, and Gender in Sociological Perspective; 2nd Edition, Boulder: Westview Press. Reprinted by China Social Science Documentation Publishing.

LEFEBVRE, Henri (1969). El derecho a la ciudad; Península, Barcelona.

MARTÍNEZ LÓPEZ, M. (2006): La participación social en el urbanismo, en los límites de la realidad. Boletín $\mathrm{CF}+\mathrm{S}$, núm. 34 .

MARTÍNEZ ROMÁN, Ma A. (2001). "Género, Pobreza y Exclusión social: Diferentes conceptualizaciones y políticas públicas", en Tortosa, J.M ${ }^{\mathrm{a}}$ (Coord.) Pobreza y Perspectiva de Género. Barcelona, Icaria, pp. 65-83.

MERINO, Mauricio (1997). "La participación ciudadana en la democracia", Cuadernos de divulgación de la cultura democrática No. 4, Instituto Federal Electoral.

PAUGAM, S. (dir.) (1996). L'exclusion, l'état des savoirs; La Découverte, París.

SEN, A (1995). Nuevo examen de la desigualdad; Alianza, Madrid.

TEZANOS, José Felix (2008). "Exclusión social, democracia y ciudadanía económica. La libertad de los iguales". Revista MTIN n. 75, p. 17-29. 
WHITEHEAD, M. (1990). The concepts and principles of equity and health. Copenhagen, Oficina Regional de la OMS para Europa (Documento número: EUR/ICP/RPD 414; http://whqlibdoc.who.int/euro/-1993/EUR_ICP_ RPD_414.pdf) [en línea, 7/5/2013]

YOUNG, I.M. (1990). La justicia y la política de la diferencia; edición de 2000, Cátedra, Madrid. 